\title{
Planar Laser-Induced Fluorescence Velocity Measurements of Retropropulsion Jets in a Mach 12 Freestream
}

\author{
Joshua R. Codoni, ${ }^{1}$ Erin M. Reed, ${ }^{2}$ and James C. McDaniel ${ }^{3}$ \\ Department of Mechanical and Aerospace Engineering, University of Virginia, Charlottesville, VA, 22903 \\ Hicham Alkandry ${ }^{4}$ and Iain D. Boyd ${ }^{5}$ \\ Department of Aerospace Engineering, University of Michigan, Ann Arbor, MI, 48109
}

\begin{abstract}
Retropropulsion is not well understood, yet is a necessary technology development for high mass Mars missions, which require new methods to adequately slow large masses from hypersonic velocities in the upper Martian atmosphere to subsonic speed to facilitate a landing configuration. In this study, hypersonic retropropulsion using a single retrorocket located at the stagnation point of a 70-deg blunt body model in a low-density Mach 12 freestream flow is investigated using planar laser-induced fluorescence, with iodine as the seeding species, to obtain planar velocity measurements. Impact shift, which typically complicates velocity measurements, is eliminated by utilizing two laser sheet angles and flowfield symmetry. Two models are examined, one with a sonic jet exit and a second with a supersonic Mach 2.66 jet exit. Flowfield geometries for both configurations are similar, with the retrorocket pushing the bow shock away from the model forebody and forming a stagnation region between the bow shock and retrorocket jet plume. The supersonic test case penetrates further upstream than the sonic case, has a thinner jet plume, and a small recirculation region just outside of the jet plume and near the forebody, which is not detected in the sonic test case. Experimental results show good agreement with CFD computed streamlines and velocity magnitude contours.
\end{abstract}

\footnotetext{
${ }^{1}$ Graduate Student, Student Member AIAA

${ }^{2}$ Graduate Student, Student Member AIAA

${ }^{3}$ Professor, Associate Fellow AIAA

${ }^{4}$ Post Doctoral Researcher, Member AIAA

${ }^{5}$ James E. Knott Professor of Engineering, Fellow AIAA
} 


\section{Nomenclature}

\begin{tabular}{|c|c|c|c|c|c|}
\hline \multirow[t]{2}{*}{$\mathrm{A}$} & \multirow[t]{2}{*}{$=$} & \multicolumn{3}{|l|}{ Empirically Determined } & \multirow{2}{*}[\mathrm{m}^{\mathrm{s}}]{} \\
\hline & & Constant & $\mathrm{S}_{\mathrm{F}}$ & $=$ & \\
\hline \multirow[t]{2}{*}{$\mathrm{A}_{21}$} & $=$ & Fluorescence Emission & $\mathrm{u}$ & $=$ & Velocity $[\mathrm{m} / \mathrm{s}]$ \\
\hline & & Rate $\left[\mathrm{s}^{-1}\right]$ & V & $=$ & Voigt Function \\
\hline $\mathrm{A}_{\mathrm{e}}$ & $=$ & $\begin{array}{l}\text { Exit Area of Propulsive } \\
\text { Deceleration Jet }\left[\mathrm{m}^{2}\right]\end{array}$ & $\mathrm{x}$ & $=$ & $\begin{array}{l}\text { Distance from Orifice } \\
{[\mathrm{mm}]}\end{array}$ \\
\hline $\mathrm{C}$ & $=$ & $\begin{array}{l}\text { Fluorescence Signal } \\
\text { Constant }\end{array}$ & $\mathrm{x}_{0}$ & $=$ & $\begin{array}{l}\text { Empirically Determinec } \\
\text { Constant [mm] }\end{array}$ \\
\hline $\mathrm{C}_{\mathrm{T}}$ & $=$ & Coefficient of Thrust & $\mathrm{x}_{\mathrm{m}}$ & $=$ & Distance to Mach Disk \\
\hline $\mathrm{D}$ & $=$ & Orifice Diameter [mm] & & & {$[\mathrm{mm}]$} \\
\hline $\mathrm{E}_{\mathrm{ij}}$ & $=$ & Strain Rate $\left[\mathrm{s}^{-1}\right]$ & $\Delta v_{\mathrm{D}}$ & $=$ & Doppler Line Width \\
\hline$f_{s}$ & $=$ & Iodine Seeding Fraction & {$[\mathrm{GHz}]$} & & \\
\hline \multirow[t]{2}{*}{$f_{v^{\prime}, J^{\prime}}$} & $=$ & Boltzmann Population & $\Delta v_{\text {Dop }}$ & $=$ & Doppler Shift [GHz] \\
\hline & & Fraction & $\Delta v_{\mathrm{I}}$ & $=$ & Impact Shift [GHz] \\
\hline I & $=$ & Laser Intensity [W] & $\Delta v_{\mathrm{T}}$ & $=$ & Total Frequency Shift \\
\hline M & $=$ & Mach Number & & & {$[\mathrm{GHz}]$} \\
\hline $\mathrm{n}$ & $=$ & Number Density $\left[\mathrm{m}^{-3}\right]$ & $\gamma$ & $=$ & Specific Heat Ratio \\
\hline $\mathrm{p}$ & $=$ & Pressure $\left[\mathrm{N} / \mathrm{m}^{2}\right]^{2}$ & $\dot{\mathrm{m}}$ & $=$ & Mass Flow Rate $[\mathrm{kg} / \mathrm{s}]$ \\
\hline $\mathrm{T}$ & $=$ & Temperature $[\mathrm{K}]$ & $\omega$ & $=$ & Vorticity $\left[\mathrm{s}^{-1}\right]$ \\
\hline$T$ & $=$ & Jet Thrust [N] & & & \\
\hline q & $=$ & $\begin{array}{l}\text { Dynamic Pressure } \\
{\left[\mathrm{N} / \mathrm{m}^{2}\right]}\end{array}$ & Subscripts & & \\
\hline \multirow[t]{2}{*}{ Q } & $=$ & Collisional Quenching & $\infty$ & $=$ & Freestream Conditions \\
\hline & & Rate $\left[\mathrm{s}^{-1}\right]$ & e & $=$ & Retrorocket Jet Exit \\
\hline $\mathrm{S}$ & $=$ & Surface Area of Aeroshell & & & \\
\hline
\end{tabular}

\section{Introduction}

$\mathrm{T}$

HE Mars Science Laboratory (MSL) marked the seventh successful landing on Mars and set new benchmarks for capabilities, such as the heaviest rover to date which was landed with the most precision. However, the upper limits of the current technology in use for landing on Mars - heritage Viking-era techniques such as a 70-deg sphere-cone blunt body and supersonic parachutes, are quickly being approached. One of the most challenging aspects of a Mars mission is the entry, descent, and landing (EDL) phase of the mission. In this portion, the lander must decelerate from hypersonic velocities to low enough speeds to facilitate a successful landing configuration. Unfortunately, the primary methods currently in use for decelerating, namely aeroshell drag and supersonic parachutes, are not as effective on Mars due to the average atmospheric density only being approximately $1 \%$ of Earth's. ${ }^{1}$

It is estimated that current capabilities, or the Viking-era techniques, along with use of aerocapture (passing through the upper atmosphere to brake before initiating EDL) can be extended to missions requiring a $1,500 \mathrm{~kg}$ payload. However, human missions to Mars will require landed masses one to two orders of magnitude larger. ${ }^{2,3}$ Therefore, it is necessary to research alternative methods for decelerating proposed future high mass Mars entry systems (HMMES). Possible methods include drag augmentation via hypersonic and supersonic inflatables (trailing ballutes, or an expandable aeroshell) as well as supersonic and hypersonic retropropulsion (SRP \& HRP), which uses retrorocket(s) placed on the aeroshell forebody to thrust opposite the direction of motion and thus decelerate the lander. The latter method is the focus of this study. SRP and HRP work initially began in the 1950s and continued through the $1970 \mathrm{~s}^{4,5}$, but was later relegated in the 1970s in favor of simply using a parachute to further slow landers down. Due to materials and sizing limits of parachutes, recently, interest has been renewed for retropropulsion and also into extending that interest beyond supersonic regimes into hypersonic regimes as well, see Refs. 6, 7, 8, 9, and 10.

Previous and renewed experimental efforts in retropropulsion have typically been limited to Schlieren/shadowgraph visualization techniques and pressure taps and surface temperature measurements, which are limited in application to understand flowfield aerodynamics. Further, all recent work, to the best of the authors' knowledge, has been for SRP applications. The following work is an extension of previous visualization and mole fraction experiments, ${ }^{11}$ and uses planar laser-induced iodine fluorescence (PLIIF) to resolve point-wise velocity measurements of a single, central HRP configuration to better understand the fundamental aerodynamics associated 
with the highly complex interaction of retropropulsion jets with a Mach 12 freestream. Two experimental models are investigated, both with a single propulsive decelerator (PD) jet located at the stagnation point on the forebody of a 70-deg blunt body cone. The first model uses an underexpanded jet and is sonic at the jet exit. The second model has a converging-diverging nozzle and is supersonic at the jet exit $(\mathrm{Mach}=2.66)$. By utilizing the non-intrusive optical PLIIF technique, it is possible to probe the flow without disturbing it and produce a flow velocity vector at each camera pixel. The high-resolution data set will then be used for validation of computational analysis.

\section{Experimental Setup}

A detailed description of the experimental facility, and general optical set-up necessary for the PLIIF experiment is discussed in Ref. 11. The continuous flow wind tunnel facility is an underexpanded jet, computationally shown in Figure 1, that produces mixed continuum/rarefied flows with Knudsen numbers (ratio of molecular mean free path to orifice diameter) approaching 1 and Mach numbers from 1 to 16 before terminating in a Mach disk. ${ }^{12}$ Nitrogen gas, seeded with approximately $200 \mathrm{ppm} \mathrm{I}_{2}$, isentropically expands through a thin plate orifice of $2 \mathrm{~mm}$ diameter into a continuously evacuated chamber. The test section size is a function of mass flow rate and vacuum system pump efficiency, or, essentially can be determined based on the stagnation pressure, orifice size, and back pressure achieved (which are $1.8 \mathrm{~atm}, 2 \mathrm{~mm}$, and approximately $280 \mathrm{mTorr}$, respectively). This results in a test section length of approximately $8 \mathrm{~cm} .{ }^{13}$ Since this underexpanded flow is well modeled, testing at a desired Mach number can be achieved by placing the test model on the jet centerline a known distance from the exit orifice. The distance

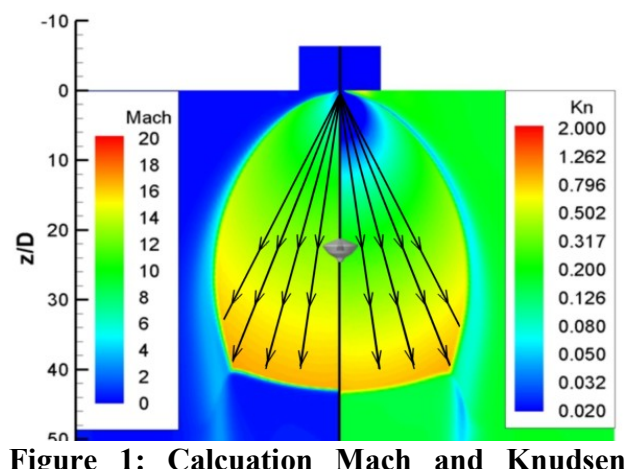

Figure 1: Calcuation Mach and Knudsen numbers in hypersonic test section ${ }^{10}$ necessary for the desired Mach number is calculated via the Ashkenas \& Sherman relationship in equation 1:

$$
M=A\left(\frac{x-x_{0}}{D}\right)^{\gamma-1}-\frac{1}{2}\left(\frac{\gamma+1}{\gamma-1}\right)\left[A\left(\frac{x-x_{0}}{D}\right)^{\gamma-1}\right]^{-1}
$$

where $\mathrm{x}_{0}$ and $\mathrm{A}$ are constants empirically determined by Ashkenas and Sherman for the specific heat ratio corresponding to $\mathrm{N}_{2}$, the test section gas.

The models in this study are a single, central PD jet models as shown in Figure 2, and a supersonic jet model. The models are constructed of aircraft aluminum, and painted matte black to limit surface reflections from the argonion laser. The frontal aeroshell diameter is $10 \mathrm{~mm}$ and the sonic model has a jet exit of $0.5 \mathrm{~mm}$, while the supersonic model has a throat of $0.5 \mathrm{~mm}$ and an exit diameter of $0.9 \mathrm{~mm}$, corresponding to a jet exit Mach of 2.66. Nitrogen seeded with $\mathrm{I}_{2}$ is supplied via a sting on the aft-body of the model. The thrust coefficient $\left(\mathrm{C}_{\mathrm{T}}\right)$, calculated by equation 2 , is used in the study to compare results with other

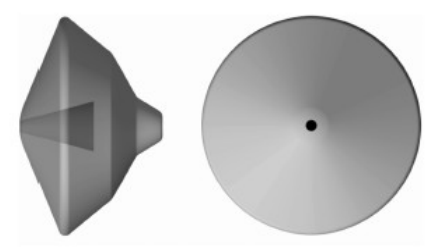

Figure 2: Sonic PD jet model experimental facilities.

$$
\mathrm{C}_{\mathrm{T}}=\frac{\mathrm{T}}{\mathrm{q}_{\infty} \mathrm{S}}=\frac{\dot{\mathrm{m}} u_{\mathrm{e}}+\left(\mathrm{p}_{\mathrm{e}}-\mathrm{p}_{\infty}\right) \mathrm{A}_{\mathrm{e}}}{\mathrm{q}_{\infty} \mathrm{S}}
$$

The $\mathrm{C}_{\mathrm{T}}$ in equation 2 is calculated using isentropic conditions and the Ashkenas and Sherman relationship (equation 1) for the freestream conditions to yield a thrust coefficient in relation to PD jet stagnation pressure, given the known and previously stated conditions of the test facility.

\section{PLIIF Experimental Method}

PLIIF is an optical, non-intrusive, time-averaged measurement technique which has been extensively used for qualitative flow visualizations ${ }^{11,14}$ and quantitative species concentration, pressure, temperature, and velocity measurements. ${ }^{11,15,16}$ It is advantageous over other methods, such as Schlieren/shadowgraph, due to the high signal level and spatial resolution in mixed rarefied/continuum flows. Another benefit of PLIIF is the ability to produce accurate measurements across shocks, unlike other methods such as particle image velocimetry. It is also preferable 
to standard pressure taps and thermocouple measurements due to the ability to obtain pressure and temperature measurements at each pixel point within the flowfield, away from the surface of the model, while not disturbing the flow.

The focus of this study is to obtain and report planar velocity measurements for a HRP flowfield. The PLIIF experiment involves seeding $\mathrm{N}_{2}$ flow with approximately $200 \mathrm{ppm} \mathrm{I}_{2}$, and using an argon-ion laser operating at $514.5 \mathrm{~nm}$ to excite discrete molecular transitions. Iodine molecules exposed to the laser radiation will be excited from the ground state to an upper energy state. The excited molecules return to the ground state via molecular collisions (quenching) or via emitting radiation (fluorescence) which is collected with a charge-coupled device (CCD) camera. The argon-ion laser can be operated in two different manners, broadband or narrowband. Broadband operation is when the laser linewidth is much greater than the $\mathrm{I}_{2}$ absorption linewidth, while for narrowband operation the laser linewidth is much less than the $\mathrm{I}_{2}$ absorption linewidth. Broadband laser operation can be used for flow visualization and species concentration measurements, while narrowband operation is necessary for pressure, temperature, and velocity measurements. However, as the focus of this paper is to obtain and analyze the velocity profile of a HRP experiment, only narrowband laser operation will be discussed.

Iodine transitions which are accessible within the argonion laser gain profile at static, room temperature, and atmospheric pressure are shown in Figure 3, for STP conditions with no relative motion. The transitions of interest in this study are the P13/R15 and P48/P103 absorption lines. With an intercavity etalon installed, the laser can be scanned through the gain profile by discrete frequency steps, and at each step an image of the flowfield is captured. By monitoring the laser frequency, and the fluorescence signal level at each individual pixel, an absorption spectrum, such as shown in Figure 4, can be compiled for each point (pixel) in the plane. The absorption spectrum depends on the thermodynamic state of the flow, as well as velocity relative to the laser sheet. The resulting fluorescence spectra can then be modeled by equation $3:{ }^{16}$

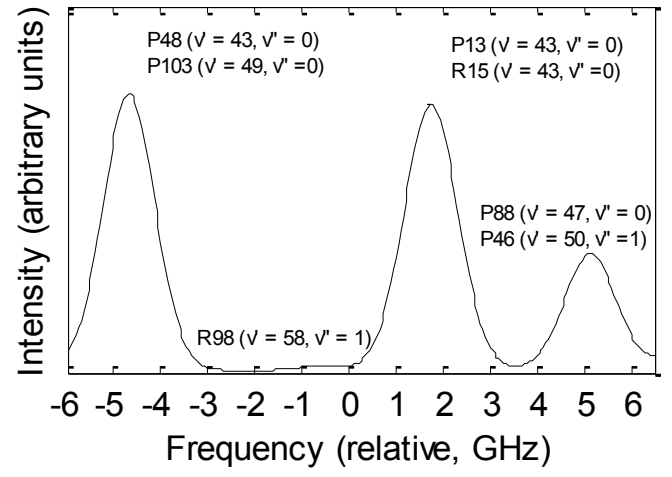

Figure 3: Iodine Absorption transitions within argonion laser gain profile

$$
S_{F}=C f_{v^{\prime \prime} J^{\prime \prime}}(T)\left[\frac{A_{21}}{A_{21}+Q(p, T)}\right] \frac{V(p, T, u, v)}{\Delta v_{D}(T)} I f_{S} n
$$

Note the Voigt function is dependent on pressure, temperature, molecular velocity, and frequency of the laser. The molecular velocity dependence is caused by the Doppler shift, where the absorbing iodine molecule interacts with the incident laser radiation at a shifted frequency due to its motion relative to the laser sheet. Molecules moving toward the laser sheet will observe the laser radiation at a higher frequency, while molecules moving away from the laser sheet will observe the radiation at a lower frequency (molecules moving perpendicular to the laser sheet will not observe a shifted spectra). The Doppler shift for velocity in the direction of the laser sheet is given by equation 4 :

$$
\Delta v_{D o p}=-\frac{u}{\lambda}
$$

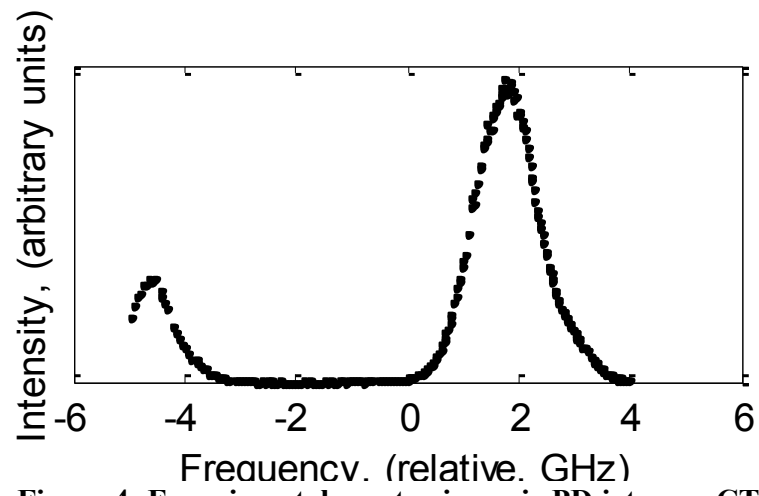

Figure 4: Experimental spectra in sonic $P D$ jet core, $C T=1.0$

In order to obtain an absolute velocity for each point (pixel), it is necessary that the Doppler shifted spectra must be compared to some reference point. The reference point for this work is obtained by splitting a portion of the laser beam to a static cell filled with $\mathrm{I}_{2}$ at a known pressure and temperature, and recording the resulting spectra with a photo multiplier tube (PMT). Recall, the velocity calculated by equation 4 is only for motion in the direction of the laser sheet. Therefore, it is necessary to use a second laser sheet to resolve a second velocity component, and thus the total planar velocity vector. 
However, there is a complication added to the process by only using two laser sheet angles and a static cell to resolve the planar velocity arises. The Doppler shift is only part of the total observed shift. The overall frequency shift is due to the Doppler shift, and impact shift, as shown in Equation 5.

$$
\Delta v_{T}=\Delta v_{D o p}+\Delta v_{I}
$$

As flows become more rarefied, the impact shift will go to zero due to fewer molecular collisions, however in higher temperature and pressure regions, the impact shift is non-negligible. Therefore to resolve the Doppler shift, and thus the velocity vector from shifted spectra, it is necessary to either calculate the impact shift with a known pressure and temperature ${ }^{17}$ or use some other method to eliminate the term. Unlike the Doppler shift, impact shift is not directiondependent, and thus can easily be cancelled by using a counter-propagating beam approach, or symmetry of the flow, if possible. By using the symmetry of the flow (about the central axis of the model) two independent laser sheet angles produce four velocity vector components, two of which are equal, but opposite. This simulates the use of counter-propagating beams, as discussed in-depth in Ref. 16. With the impact shift accounted for using symmetry, then, it is simply necessary to compare each laser angle to the reference point obtained from obtaining the static-velocity spectra in a static cell at known temperature and pressure (with negligible impact shift) in order to obtain the two dimensional planar velocity at each point in the flowfield.

The analysis of experimental data is performed using Matlab's global optimization toolbox. Essentially a nonlinear least squares fit of the theoretical model to the experimental data is performed. Once the data is fit, the peak frequency of the shifted spectra is obtained and can be compared to the static cell. Finally, the impact shift is cancelled and the velocity vector is resolved for each camera pixel.

\section{Computational Fluid Dynamics}

Experimental results will be compared with numerical simulations executed using LeMANS, a parallelized CFD code for simulating hypersonic reacting flows. ${ }^{18,19,20}$ LeMANS solves the laminar three-dimensional Navier-Stokes equations on unstructured computational grids, including thermo-chemical nonequilibrium effects. The transport properties can be calculated using several options. For this study, mixture transport properties are calculated using Wilke's semi-empircal mixing rule with species viscosities calculated using Blottner's model and species thermal conductivities determined using Eucken's relation. The finite-volume method applied to unstructured grids is used to solve the set of partial differential equations. Time integration is performed using a point implicit or line implicit method.

The flow is modeled assuming that the continuum approximation is valid ${ }^{21}$. Furthermore, for this work it is assumed that the translational and rotational energy modes of all species can be described by two different temperatures, $\mathrm{T}_{\mathrm{Tra}}$ and $\mathrm{T}_{\text {Rot }}$ while the vibrational and electronic energy modes of all species are frozen at the stagnation value. In order to accurately simulate the flow in the experimental facility, $\mathrm{I}_{2}$-seeded $\mathrm{N}_{2}$ gas is used in the numerical simulations with a seeding ratio of $200 \mathrm{ppm}$. In the freestream, the rotational temperature is assumed to be equal to the translational temperature. Also, the Ashkenas and Sherman conditions are used as flow conditions input to LeMANS at the upstream boundary.

\section{Results}

The PLIIF technique has been applied to two flowfields of interest, a single central sonic PD jet model, and a single central supersonic PD jet model, in Mach 12 freestream flow. Flowfield velocity magnitude and streamlines are shown in Figure 5a for the sonic test case for $\mathrm{C}_{\mathrm{T}}=1.0$. Freestream flow is from top to bottom and the retrorocket jet exit is centered at $\mathrm{Y} / \mathrm{D}$ and $\mathrm{X} / \mathrm{D}$ of zero, where $\mathrm{D}$ corresponds to model diameter of $1 \mathrm{~cm}$. The retrorocket expands from the jet exit to a terminal shock, after which the flowfield slows to subsonic velocities. The freestream flow forms a normal shock over this subsonic region, and also decelerates to subsonic speeds. In the subsonic region, the retrorocket fluid and freestream fluid mix, turn downstream and accelerate as the fluid flows towards the model shoulder. The supersonic test case is shown in Figure 5b, and overall exhibits similar flowfield properties to the sonic test case. Differences between the sonic and supersonic flowfields are evident in the retrorocket jet structure. For example, the sonic jet plume does not penetrate as far upstream as the supersonic case and has a more rounded structure due to the higher pressure at the sonic jet exit which causes the jet to expand more in the radial direction. Further, the stagnation region between the retrorocket structure and freestream bow shock is slightly larger for the sonic jet than the supersonic jet, likely due to the more rounded structure of the sonic jet plume which is not as oblique as the supersonic jet plume. Another major difference in the flowfield structures appears near the base of 
the supersonic jet plume, outside of the barrel shock structure, where the supersonic flowfield has a subsonic recirculation region, which is not evident in the sonic test case.

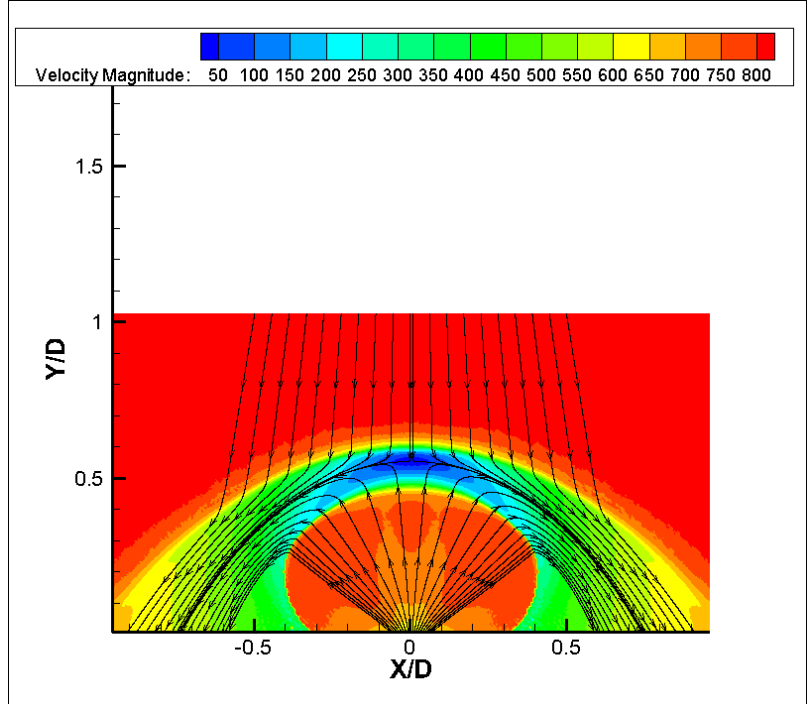

(a) Sonic retrorocket, $\mathrm{M}_{\mathrm{Jet}}=1.0$

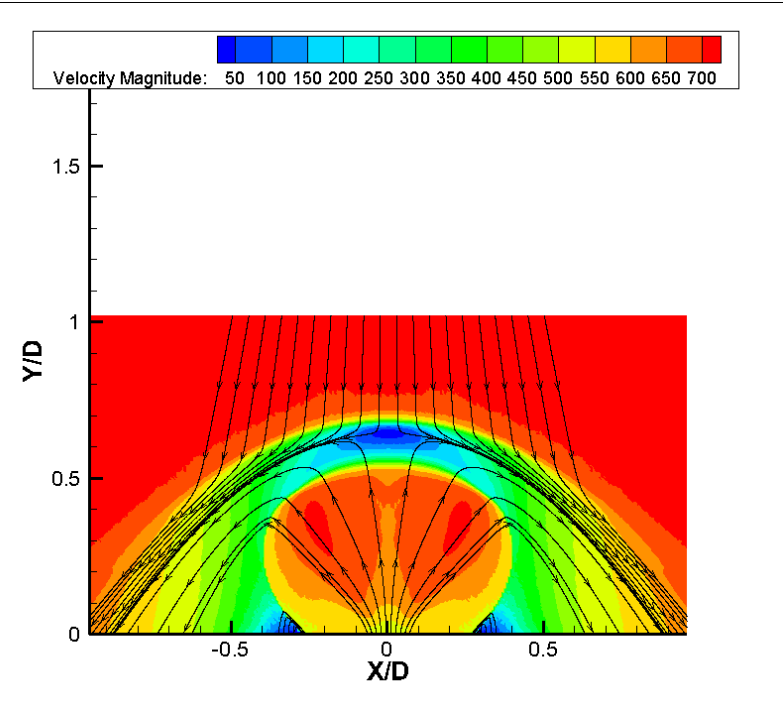

(b) Supersonic retrorocket, $\mathrm{M}_{\text {jet }}=2.66$

Figure 5: Velocity magnitude in $\mathrm{m} / \mathrm{s}$ and streamlines

With the spatially resolved planar velocity field it is possible to calculate a two dimensional strain tensor, which is given by equation 6 .

$$
E_{i j}=\frac{1}{2}\left(\frac{\partial u_{i}}{\partial j}+\frac{\partial u_{j}}{\partial i}\right)
$$

The resulting analysis for the sonic test case is shown in Figure 6(a), 6(b) and 6(c), where $E_{x x}$ and $E_{y y}$ correspond to expansion (positive values) or compression (negative values) in the respective $\mathrm{x}$ and $\mathrm{y}$ directions and the offdiagonal terms $\mathrm{E}_{\mathrm{xy}}$ and $\mathrm{E}_{\mathrm{yx}}$ correspond to shearing strains. Once again, freestream flow is from top to bottom, and the retrorocket nozzle exit is located at $\mathrm{X} / \mathrm{D}$ and $\mathrm{Y} / \mathrm{D}$ of 0 . Note, only half of the flowfield is shown in these images. It is apparent there is a strong horizontal compression in the retrorocket jet plume boundary and slight horizontal expansion in the bow shock region. Whereas in the vertical direction there is a strong vertical compression in the bow shock as well as slightly aft of the bow shock in the stagnation region, and slight vertical expansion in the retrorocket jet plume boundary. As expected, a strong shearing effect is evident in the retrorocket jet boundary where the fluid flow just outside of the jet plume boundary is moving relatively slowly, and in the opposite direction of the high speed retrorocket jet fluid. This shearing force is responsible for the recirculation region which tends to develop near the model shoulder at lower $\mathrm{C}_{\mathrm{T}}$, when the jet expansion is not as large. However, the recirculation region is not visible for the sonic test case, but can be noted for the supersonic case in Figure 5(b). This relationship will be discussed in detail when comparing with the CFD results. It is also possible to calculate flow vorticity as defined by the following equation:

$$
\omega_{z}=\frac{\partial u_{y}}{\partial x}-\frac{\partial u_{x}}{\partial y}
$$

Vorticity for the sonic retrorocket test case is shown in Figure 6(d). The greatest vortices are located in the shearing region on the retrorocket jet plume boundary where the mixed retrorocket and freestream fluid is flowing downstream as the retrorocket jet fluid is expanding upstream. Another notable area of relatively strong vortices is in the bow shock region where the freestream fluid makes an abrupt turn as it flows around the model and proceeds downstream. For brevity, only the sonic test results are shown herein, however the supersonic test results for the strain tensor matrix and voriticity overall display the same trends as for the sonic case. 


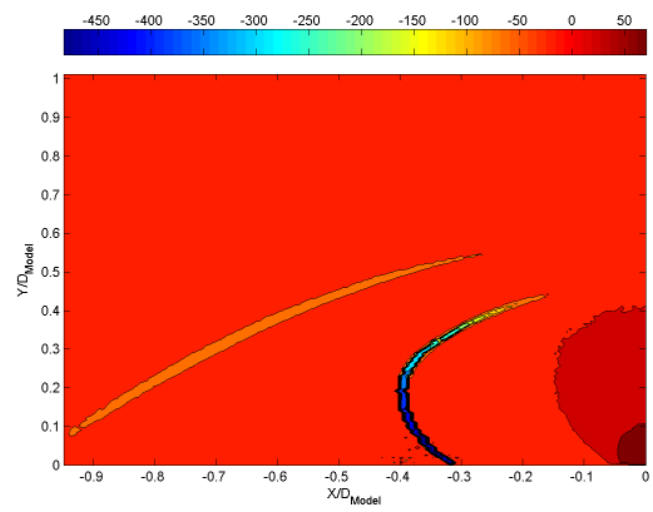

(a) $E_{x x}$

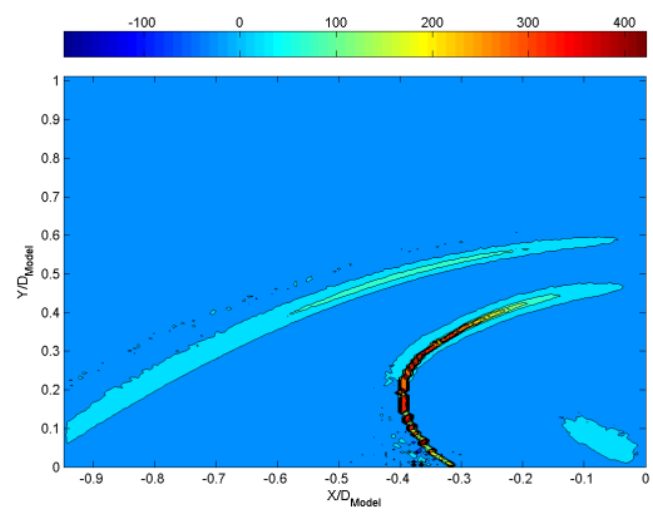

(c) $E_{x y}$

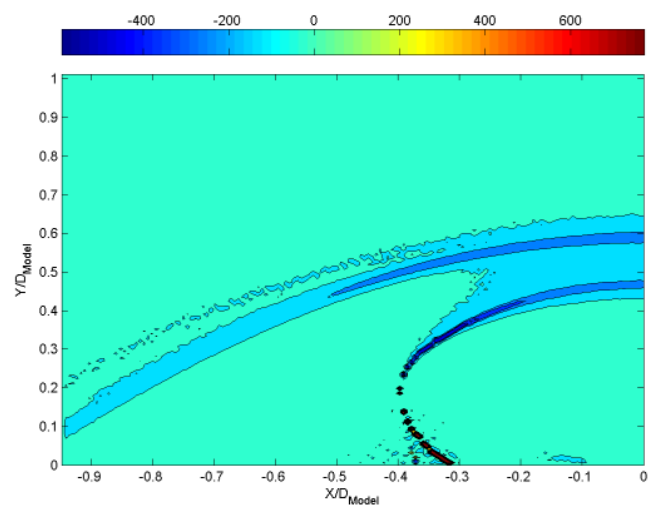

(b) $E_{\mathrm{yy}}$

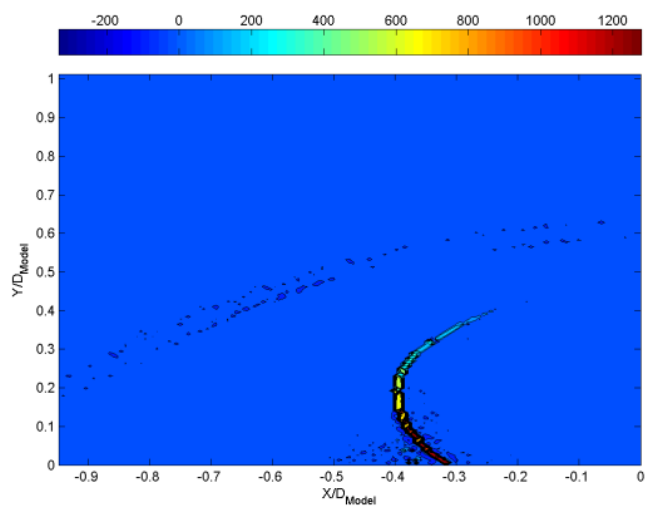

(d) $\omega_{z}$

Figure 6: Components of strain tensor for sonic retrorocket, $M_{\text {Jet }}=1.0$

Due to the retrorocket behavior being identical to the freestream flow created by a freejet expansion, it is possible to estimate the total pressure at the stagnation point between the retrorocket Mach disk and bow shock with equation 8 from Ashkenas and Sherman. ${ }^{13}$

$$
p_{1}=p_{0}\left(\frac{0.67 D_{J e t}}{x_{m}}\right)^{2}
$$

By observing where the fluid velocity begins to decelerate, it is possible to identify the location of the retrorocket Mach disk, evident in Figure 8 to be 8.7 jet exit diameters upstream. Knowing this distance and the retrorocket plenum stagnation pressure, 299.9 Torr, equation 8 is used to calculate the stagnation pressure between the Mach disk and bow shock, $\mathrm{p}_{1}$, to be 1.8 Torr. This computed value can be further confirmed by calculating the stagnation pressure aft of the freestream bow shock, which is equal to $p_{1}$. Because the retrorocket pushes the bow shock farther upstream it is necessary to estimate the location of the bow shock in relation to the model stagnation point, which is positioned at the location corresponding to Mach 12 freestream flow, to estimate the Mach number at the bow shock location. Estimating the bow shock location in the same manner as the Mach disk location, the bow shock is estimated to be in Mach 11.20 flow. Given the freestream plenum stagnation pressure is 1,370 Torr and assuming a normal shock, this corresponds to a post-shock stagnation total pressure of 2.4 Torr, which is in excellent agreement with the value predicted by the Ashkenas and Sherman relationship, equation 8.

Experimental velocity magnitude and streamline results are compared with LeMANS CFD solutions in Figure 8. In figure 8 the freestream flow is from left to right and the jet exit nozzle is located at X/D and Y/D of 0 . The CFD results are shown for positive Y/D values, and the experimental results are negative Y/D. Overall, there is good agreement between the experimental and computational results. Slight discrepancies are notable with the shock stand-off distance (point where the bow shock begins), bow shock thickness, retrorocket jet plume size, and 
recirculation region near the bottom of the jet plume. The CFD computations indicate a larger shock stand-off distance and stagnation region for both the sonic and supersonic cases while the experimental results show an overall larger retrorocket jet plume structure, wider as well as longer, and a thicker bow shock. Discrepancy between the experiment and CFD in the bow shock thickness could be due to non-continuum effects in this area, which will not be well predicted by CFD. The larger jet plume size for the experimental results serves as an explanation for the lack of recirculation region noted in the sonic experimental test case, and smaller recirculation region for the supersonic test case when compared with the CFD results. As noted in reference 20, the largest recirculation regions are expected for the lower $\mathrm{C}_{\mathrm{T}}$. As $\mathrm{C}_{\mathrm{T}}$ increases, and thus jet plume size, the recirculation is pushed farther towards the model shoulder until finally not being evident around $\mathrm{C}_{\mathrm{T}}$ of 2.0. The larger jet plume size seen in experimental results would then suggest a smaller recirculation region and explain why it may not be visible for the sonic test case. Further, a small recirculation region is evident for the supersonic test case, where the jet plume is longer but not quite as wide, which would cause the recirculation region to be larger. Therefore, while the experimental and CFD results differ slightly, the same overall trends are evident.

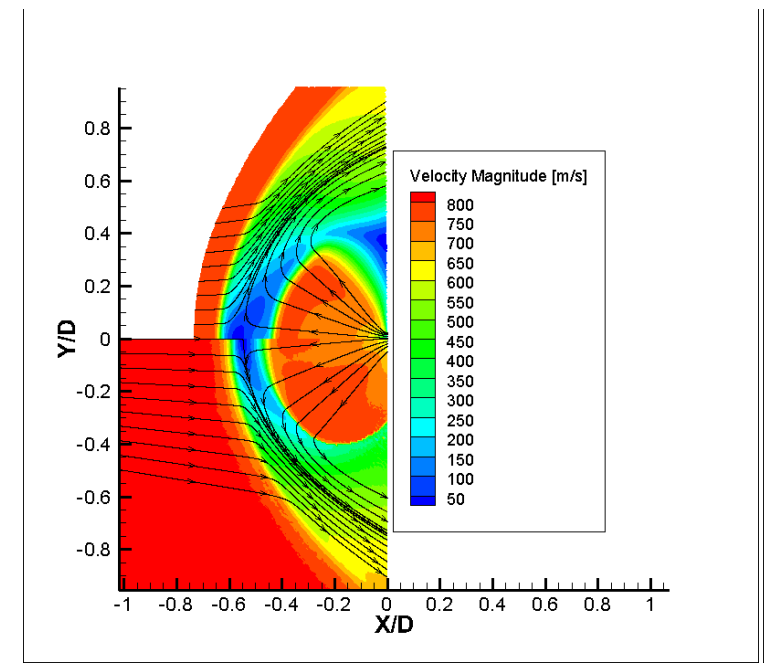

(a) Sonic jet, $\mathrm{M}_{\mathrm{Jet}}=1.0$

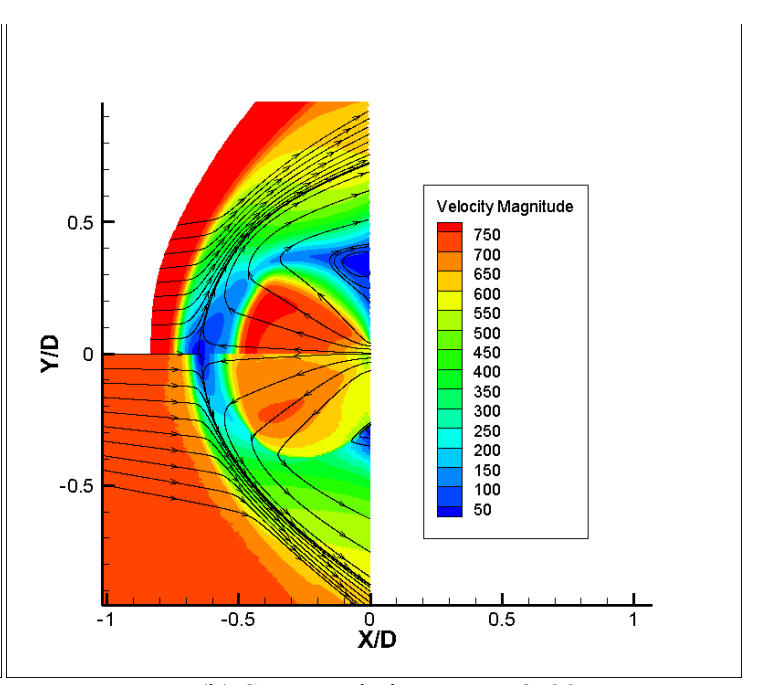

(b) Supersonic jet, $\mathrm{M}_{\text {Jet }}=2.66$

Figure 8: Comparison of CFD (top) to experimental (bottom) results

\section{Conclusion}

Planar laser-induced iodine fluorescence experimental velocity measurements have been performed for two different retropropulsion models: one with a sonic propulsive decelerator jet located on the stagnation point of a 70deg. blunt cone, and the second with the same configuration, but with a supersonic propulsive decelerator jet, with a jet exit of Mach 2.66. Velocity measurements of the sonic and supersonic models for coefficient of thrust equal to one reveal the sonic test case to have a more broad jet structure that does not penetrate as far upstream as the supersonic test case. It has been confirmed that a free stagnation region forms between the retrorocket Mach disk and bow shock, which is pushed upstream of the model forebody by the retrorocket. Further, the supersonic test case shows a recirculation region near the base of the jet plume (between the model shoulder and jet exit), whereas there is no recirculation region evident for the sonic test case.

As expected, strain tensor calculations confirm a vertical compression where the bow shock is located above the free stagnation region, and a large horizontal compression in the retrorocket jet plume. The retrorocket jet plume induces a large shear on the fluid aft of the bow shock region, which is flowing opposite the direction of the retrorocket, and hence the largest flow vortices are also detected in this region of the flow. Regions of small shear and vorticity are also seen in the bow shock region where the freestream fluid abruptly turns to flow around jet plume and model shoulder. Experimental planar velocity distributions were also used to locate the precise location of the bow shock and retrorocket Mach disk, which makes it possible to use shock-jump conditions and the Ashkenas and Sherman relationships to compute the expected stagnation pressure between the bow shock and retrorocket Mach disk. Expected stagnation pressure in this region is estimated to be 2.4 Torr from the shock jump 
condition in Mach 11.2 flow (which corresponds to the Mach number the bow shock is pushed to by the retrorocket jet plume) and 1.8 Torr from the Ashkenas and Sherman relationship, which are in excellent agreement.

Finally, experimental planar velocity distributions have been compared with computational fluid dynamics. Overall, the computational fluid dynamic results compare well with the experimental velocity distribution. Some discrepancies are visible in the retrorocket jet penetration, the distance the retrorocket travels into the freestream flow, retrorocket jet plume width, and the lack of a recirculation region for the sonic jet test case, and a smaller recirculation region for the supersonic test case. Although, the wider retrorocket plume in conjunction with the lack of a recirculation region for the sonic test case, as well as smaller recirculation region for the supersonic test case, agree with the flowfield trends of the computational fluid dynamic results. As the jet plume becomes wider the recirculation region is pushed farther towards the model shoulder, until eventually not being evident at the thrust coefficient of 2.0. Further, the recirculation region is larger for supersonic computational results because the jet plume is not as wide near the retrorocket nozzle exit, which agrees well with the trends displayed in the experimental test cases at thrust coefficient of 1.0.

\section{References}

${ }^{1}$ Braun, R. D., and Manning, R. M., "Mars Exploration Entry, Descent, and Landing Challenges," Journal of Spacecraft and Rockets, Vol. 44, No. 2, 2007, pp. 310-323.

${ }^{2}$ Zang, T. A., and Munk, M. M., et al., "Entry, Descent and Landing Systems Analysis Study: Phase 1 Report," NASA TM 2010-216720, July 2010.

${ }^{3}$ Steinfeldt, B. A., Theisinger, J. E., Korzun, A. M., Clark, I. G., Grant, M. J., and Braun, R. D., "High Mass Mars Entry, Descent, and Landing Architecture Assessment," AIAA Paper 2009-6684, Sept. 2009.

${ }^{4}$ Jarvinen, P. O., and Adams, R. H., "The Aerodynamic Characteristics of Large Angled Cones with Retrorockets," NASA CR NAS 7-576, Feb. 1970.

5 Keyes, J.W. and Hefner, J.N., "Effect of Forward-Facing Jets on Aerodynamic Characteristics of Blunt Configurations at Mach 6," Journal of Spacecraft and Rockets, Vol. 4, No. 4, 1967, pp. 533-534.

6 Adler, M., Wright, M., Campbell, C., Clark, I., Engelund, W., Rivellini, T., "Entry, Descent, and Landing Roadmap," NASA TA09, April, 2012.

${ }^{7}$ Edquist, K. T., Dyakonov, A. A., Korzun, A. M., Shidner, J. D., Studak, J. W., Tigges, M. A., Kipp, D. M.,

Prakash, R., Trumble, K. A., Dupzyk, I. C., "Development of Supersonic Retro-

Propulsion for Future Mars Entry, Descent, and Landing Systems,” AIAA Paper 2010-5046, June 2010.

${ }^{8}$ Korzun, A. M., and Braun, R. D., "Performance Characterization of Supersonic Retropropulsion for High-Mass Mars Entry Systems," Journal of Spacecraft and Rockets, Vol. 47, No. 5, pp. 836-848, 2010.

${ }^{9}$ Berry, S.A., Laws, C.T., Kleb, W.L., Rhode, M.N., Spells, C., Mccrea, A.C., Trumble, K.A., Schauerhamer, D.G., Oberkampf, W.L., "Supersonic Retro-Propulsion Experimental Design for Computational Fluid Dynamics Model Validation,” IEEE Aerospace Conference Paper No.1499, March 2011.

10 Palaszewski, B. and Bencic, T., "Mars Entry, Descent and Landing: Experiments with Multiple Engine Supersonic Retro Propulsion Configuration,” AIAA 2010-6594, Joint Propulsion Conference, Nashville, TN, July 2010.

${ }^{11}$ Codoni, J.R., Reed, E.M., McDaniel, J.C., Alkandry, H., Boyd, I.D., "Investigations of 4 Peripheral Propulsive Deceleration Jets on a Mars science Laboratory Aeroshell," submitted to the Journal of Spacecraft and Rockets, July, 2012.

${ }^{12}$ Staack, D., McDaniel, J.C., Glass C.E., and Miller C., "Experimental Study of Interacting Rarefied and continuum Flows." AIAA Paper 2001-2762, June 2001.

${ }^{13}$ Ashkenas, H. and Sherman, F.S., "The Structure and Utilization of Suerpsonic Free Jets in Low Density Wind Tunnels," Rarefied Gas Dynamics, Vol. 2, Academic Press, New York, 1966.

${ }^{14}$ Reed, E., Codoni J., McDaniel, J.C., Alkandry, H., Boyd, I.D., "Investigation of the Interactions of Reaction Control Systems with Mars Science Laboratory Aeroshell," AIAA Paper 2010-1558, January 2010.

${ }^{15}$ Hartfield, R.J., Hollo, S.D., and McDaniel, J.C., "Planar Temperature Measurement in Compressible Flows Using Laser-Induced Iodine Fluorescence," Optics Letters, Vol. 16, No. 2, 1991.

${ }^{16}$ Hiller, B., and Hanson, R.K., "Simultaneous planar measurements of velocity and pressure fields in gas flows using laser-induced fluorescence," Applied Optics, Vol. 27, No. 1, 1988. 
${ }^{17}$ Fletcher, D.G., "Spatially Resolved, Nonintrusive Measurements in Nonreacting Scramjet Combustor Using Laser-Induced Iodine Fluorescence," PhD Dissertation, Department of Mechanical and Aerospace Engineering, University of Virginia, January 1989.

${ }^{18}$ Scalabrin, L.C. and Boyd, I.D., "Numerical Simulation of Weakly Ionized Hypersonic Flow for Reentry Configurations,” AIAA Paper 2006-3773, June 2006.

${ }^{19}$ Martin, A., Scalabrin, L.C., and Boyd, I.D., "High Performance Modeling of Atmospheric Re-entry Vehicles," Journal of Physics: Conference Series, Vol. 341, 2012, Article 012002.

${ }^{20}$ Alkandry, H., Boyd, I.D., Reed, E.M., Codoni, J.R., and McDaniel, J.C., "Interactions of SingleNozzle Sonic Propulsive Deceleration Jets on Mars Entry Aeroshells," AIAA Paper 2010-4888, June, 2010

${ }^{21}$ Reed, E., Codoni J., McDaniel, J.C., Alkandry, H., Boyd, I.D., "Investigation of the Interactions of Reaction Control Systems with Mars Science Laboratory Aeroshell," AIAA Paper 2010-1558, January 2010. 